\title{
Escala ZICAP II: evaluación de alienación parental en niños de 9 a 15 años de padres separados en Chile
}

\section{ZICAP II Scale: Parental Alienation Assessment in 9 to 15 years-old children of separated parents in Chile}

\section{Escala ZICAP II: avaliação da alienação parental em crianças de 9 a 15 anos de pais separados no Chile}

\author{
Nelson Zicavo Martínez' ${ }^{1}$ ORCID 0000-0002-2107-8646 \\ Ricardo Rey Clericus ${ }^{2}$ ORCID 0000-0002-8583-050X \\ Luciano Ponce ${ }^{3}$ ORCID 0000-0002-7799-7473 \\ 12 Universidad del BíoBío. Chile. \\ ${ }^{3}$ Universidad Nacional de Córdoba. Argentina.
}

\begin{abstract}
Resumen: El objetivo de esta investigación fue construir un instrumento para evaluar Alienación Parental (AP) en niños y adolescentes chilenos, entre 9 y 15 años. Se confeccionó una herramienta para recoger evidencias de obstrucción al vínculo hijo/padre, lo que constituye maltrato infantil intrafamiliar grave, observado en procesos de divorcios conflictivos. Los ítems fueron construidos a partir de la conceptualización teórica de AP y se sometieron a consideración de jueces expertos quienes los enriquecieron, después de lo cual fueron sometidos a análisis estadísticos y ajustes sucesivos. De dicho proceso se obtuvo un instrumento de 29 ítems, 8 componentes y dos dimensiones (Dimensión I Alfa de Cronbach de .777, Dimensión II con un Alfa de Cronbach de .884). Esta escala se aplicó a 1181 hijos de padres separados, de 9 a 15 años, del centro sur de Chile. Se realizó el Análisis Factorial Confirmatorio sobre la estructura teórica previamente propuesta observándose valores de ajuste aceptables.
\end{abstract}

Palabras Clave: alienación parental, propiedades psicométricas, escala, maltrato infantil

Abstract: The aim of this research was to build a valid and reliable instrument to evaluate Parental Alienation (PA) in Chilean kids, between 9 and 15 years. This project started by the need of an instrument to collect evidence of child/parent bond obstruction, a source of severe intra-family child abuse observed in conflictive divorce situations. The items were constructed based on theoretical conceptualization of PA and submitted to expert's judges who agreed to review and enriched them, to be later submitted to statistical analysis and successive adjustments. The final outcome consists of 29 items, 8 components and two dimensions instrument (Dimension I: Alpha value of .777, Dimension II: Alpha value of .884). This scale was applied to a sample of 1.181 children from 9 to 15 years-old with separated parents, of the south-central regions of Chile. Confirmatory Factor Analysis was performed on the theoretical structure previously proposed, observing acceptable adjustment values.

Key Words: parental alienation, psychometric properties, scale, child abuse 
Resumo: O objetivo desta pesquisa foi construir um instrumento para avaliar a Alienação Parental (AP) em crianças e adolescentes chilenos, entre 9 e 15 anos. Foi criada uma ferramenta para coletar evidências de obstrução do vínculo filho / pai, que constitui maltrato infantil intrafamiliar grave, observado em processos de divórcio conflituoso. Os itens foram construídos a partir da conceituação teórica da AP e foram submetidos à consideração de juízes especialistas que os enriqueceram, em seguida foram submetidos a análises estatísticas e ajustes sucessivos. A partir desse processo, foi obtido um instrumento com 29 itens, 8 componentes e duas dimensões (Dimensão I, Alfa de Cronbach de .777, Dimensão II com Alfa de Cronbach de .884). Essa escala foi aplicada a 1181 filhos de pais separados, de 9 a 15 anos, do centro-sul do Chile. A Análise Fatorial Confirmatória foi realizada sobre a estrutura teórica proposta previamente, observando-se valores de ajuste aceitáveis.

Palavras-chave: alienação parental, propriedades psicométricas, escala, maltrato infantil

Recibido: 11/05/2020

Aceptado: 03/12/2020

Cómo citar:

Zicavo Martínez, N., Rey Clericus, R., y Ponce, L. (2021). Escala ZICAP II: evaluación de alienación parental en niños de 9 a 15 años de padres separados en Chile. Ciencias Psicológicas, 15(1), e-2159. doi: https://doi.org/10.22235/cp.v15i1.2159

Correspondencia: Nelson Zicavo Martínez. Universidad del BíoBío, Chile. E-mail: nzicavo@ubiobio.cl

La familia tradicional ha ido dando paso a nuevas estructuras con actuales estilos relacionales, condicionadas por la creciente independencia económica de la mujer y la progresiva participación de varones en la crianza de sus hijos y en labores domésticas. No obstante, la práctica cultural, social y jurídica indica, que ante a un divorcio o separación conyugal generalmente la mujer debe hacerse cargo de la crianza y educación de los niños, dejando atrás la posibilidad que ambos padres continúen con la tarea de paternar (Lesme \& Zicavo, 2014).

Con el divorcio suele disociarse la estructura del sistema familiar y uno de los padres suele quedar en situación asimétrica, periférica y ajena a la dinámica que antes integrara, amenazando su rol y trayendo consecuencias de impedimento relacional con sus hijos quienes pierden un referente identitario trascendente en su crecimiento, pudiendo desarrollar una variedad de trastornos mentales, que van desde un trastorno de adaptación hasta un trastorno depresivo mayor (Bernet, Wamboldt \& Narrow, 2016). Ante un divorcio altamente conflictivo, se normaliza la distancia parental masculina naturalizando las situaciones que obstaculizan la participación paterna en la crianza de sus hijos (Harman, Biringen, Ratajack, Outland \& Kraus, 2016; Zander, 2012). Adicionalmente, las conductas de AP causan dilemas morales en los niños a los que se les pide que vayan en contra de sus valores y creencias -espiar o guardar secretos- lo cual tiene consecuencias imprevisibles en el desarrollo infantil (Baker \& Verrocchio, 2016).

Por su parte, las personas encargadas de impartir justicia deben discernir cuál de los padres es idóneo para "quedarse" con los niños, decisión a menudo compleja y donde no siempre todas las partes son debidamente escuchadas (Bernet et al., 2016). Padres y madres asisten a una batalla sin fin, donde los peritos ayudan a discernir verdades de falsedades, injurias y fechorías, asuntos muy difíciles de probar (Kelly \& Johnston, 2001). 
En la actualidad no existe una prueba válida en español que demuestre la presencia del proceso de AP desde la percepción de los niños, niñas y adolescentes. No se cuenta con una herramienta especializada que permita descubrir y revelar el maltrato de que están siendo objeto los niños y algunos adultos. Tal es el propósito final de este trabajo, visibilizar la presencia del proceso de maltrato y violencia intrafamiliar a través de una escala que mida AP de manera eficiente. Además, develar su incidencia en la sociedad actual que ha dejado de ver el divorcio como el causante de patologías y disolución social. El divorcio parece no ser el problema, sino la posibilidad de que pueda desnivelar los antiguos equilibrios familiares, empoderando a unas personas más que otras con el resultado indeseable de perjudicar a los niños, a quienes la familia y la sociedad debieran proteger.

\section{Leyes y divorcio conflictivo}

La ley de violencia 20.066 (2005) en Chile, intenta regular y sancionar los actos de violencia en la familia, la define como:

"todo maltrato que afecte la vida o la integridad física o psíquica de quien tenga o haya tenido la calidad de cónyuge del ofensor o una relación de convivencia con él; o sea pariente por consanguinidad o por afinidad en toda la línea recta o en la colateral hasta el tercer grado inclusive, del ofensor o de su cónyuge o de su actual conviviente. También habrá violencia intrafamiliar cuando la conducta referida en el inciso precedente ocurra entre los padres de un hijo común, o recaiga sobre persona menor de edad, adulto mayor o discapacitada que se encuentre bajo el cuidado o dependencia de cualquiera de los integrantes del grupo familiar (Art. 5. ley 20.066)".

A dicha ley se le realizaron modificaciones (ley $\left.\mathrm{N}^{\circ} 20.480\right)$ incluyendo el femicidio como acto violento punible (2010) y aumentando las penas; no obstante, quedan espacios de atención no abordados en el ámbito de la violencia intrafamiliar, sobre todo aquella ejercida como obstrucción de vínculos entre hijos y padres posterior al divorcio de la pareja.

Durante el año 2013 se aprueba la Ley N²0.680, conocida como Ley Amor de Papá que introduce el principio de corresponsabilidad, en virtud del cual ambos padres, vivan juntos o separados, participarán en forma activa, equitativa y permanente en la crianza y educación de sus hijos (art. 224 inc. $1^{\circ}$ del Código Civil); agregando que el cuidado personal compartido estimula la corresponsabilidad de ambos padres que viven separados, en la crianza y educación de los hijos comunes (art. 225 inc. $2^{\circ}$ del CC); disminuyendo las asimetrías en la tuición de hijos de padres separados y limitando los impedimentos de contacto en divorcios conflictivos; teniendo en cuenta que cuando tiene lugar este tipo divorcio se observa la obstaculización de uno de los padres a que el otro se relacione con cercanía física y emocionalmente con sus hijos, reiteradas disputas entre los excónyuges, habitualmente presenciadas por los hijos y al menos un miembro de la pareja parental presenta la necesidad inminente de ganar y denigrar al otro (Folberg \& Milne, 2005; Zicavo, Celis, González \& Mercado, 2016).

El ejercicio de la parentalidad y los procesos de obstrucción de esta (limitación, impedimento o entorpecimiento de contacto de uno de los padres con los hijos) están fuertemente condicionados por relaciones de poder, parte esencial de la interacción humana (Ponce, Arrieta, Jalile y Guini, en Zicavo, 2016). El fin de la obstrucción de contacto vincular se alcanza cuando se perfila la presencia de Alienación Parental sobre los hijos de padres separados. Al respecto R. Gardner propone el síndrome de alienación parental (SAP), definido como un trastorno caracterizado por el conjunto de síntomas que resultan del proceso por el cual un progenitor transforma la consciencia de los hijos con el objetivo de impedir, obstaculizar o destruir los vínculos con el otro padre (Gardner, 1998). Surge principalmente en el contexto de las disputas por la guarda o custodia de los niños y su primera manifestación es una campaña de difamación en contra de uno de los padres, conscientemente estructurada por el progenitor custodio y respaldada por parte del hijo, campaña que carece de justificación (Gardner, 1998). Para este autor 
la intensidad del proceso de alienación evidenciado en los niños se puede diferenciar tipológicamente en tres niveles en aumento gradual: Leve, Moderado y Severo (abordados más adelante).

\section{Miradas Críticas Sobre Alienación Parental (AP)}

El SAP no es totalmente aceptado por el mundo académico y científico, aunque es ampliamente usado en ambientes jurídicos por disputas de padres y ante situaciones de divorcios altamente conflictivos. Tampoco la denominación de "síndrome" concita acuerdos amplios ya que autores como Bernet y Baker (2013) aun compartiendo el fondo del planteo de Gardner, se distancian de esta definición. Ellos señalan:

"Nuestra definición de AP es una condición mental en la que un niño, generalmente cuyos padres están involucrados en una separación o divorcio de alto conflicto, se alía fuertemente con uno de los padres (el padre preferido) y rechaza una relación con el otro padre (el padre alienado) sin una justificación legítima. La AP presenta un comportamiento anormal e inadaptado (negativa a tener una relación con un padre amoroso) que es impulsado por un estado mental anormal (la falsa creencia de que el padre rechazado es malvado, peligroso o indigno de amar)" (Bernet \& Baker, 2013, p. 99).

Sugieren los autores antes citados la reformulación del modelo de alienación de Gardner desmarcándose de la denominación de síndrome y proponiendo asumir los términos AP como un proceso relacional, en los que existirían fuertes contrariedades, conflictos, entre padres e hijos en el transcurso de cuidados y tutela mientras crecen. Ven con cercana identidad conceptual las enunciaciones de Kelly y Johnston (2001) quienes definen el modelo de alienación con un esquema de comportamientos infantiles caracterizados por el odio, miedo y rechazo injustificado y desproporcionado contra el padre alejado (Kelly \& Jonhston, en Bernet \& Baker, 2013). Tales conductas señaladas, no serían una enfermedad mental, más bien son evidencias de un problema relacional o de interacción familiar disfuncional (Bernet \& Baker, 2013; Siracusano, Barone, Lisi \& Niolu, 2015).

Autores como Moses y Townsend (2011), O'Donohue, Benuto y Bennett (2016), Pepiton, Alvis, Allen y Logid (2012), y Zirogiannis (2001) centran sus esfuerzos en contribuir al debate científico señalando que no existiría la suficiente evidencia empírica para que el SAP sea un diagnóstico psiquiátrico y por ende deba ser incluido en los DSM y CIE. Las publicaciones de Gardner también son cuestionadas, pues la mayoría de ellas no aparecen en revistas con referato de pares y serían de su propia editorial, por lo que dichos trabajos no fueron sometidos a revisión por pares y esto restaría el respaldo debido a sus contribuciones (Moses \& Townsend, 2011; Pepiton et al., 2012).

Por otra parte, Walker, Brantley y Rigsbee, 2004 (en O'Donohue et al., 2016) argumentaron que algunos niños presentan ansiedad de separación cuando sus padres se están divorciando. Por lo tanto, tales "síntomas" del niño pueden también demostrar sus intentos para manejar el estrés de la situación actual, independientemente de que cualquiera de los padres sea un mal actor. Además, señalan que tales síntomas pueden también ser resultado de un abuso físico o sexual (o negligencia) ocurridos antes al niño, que pueden haber sido una de las razones precipitantes del divorcio y esto en sí mismo genera confusiones inevitables y falta de rigor en el diagnóstico. Se referencia entonces un conjunto de argumentos muy detallados mediante los cuales pretenden demostrar que no hay evidencias de que las características implicadas en el SAP constituyan un síndrome válido (O'Donohue et al., 2016). Aun así, se asume por estos autores que en el abordaje de este complejo asunto, si bien no debiera rescatarse el concepto SAP por las implicaciones forenses que pudiera tener, resulta necesario comprender que en no pocas oportunidades algunos padres se involucran en comportamientos específicos, con el fin de dañar la relación del hijo con el otro padre. 
Los manuales de clasificaciones DSM y CIE no solo cuentan con el debido respaldo de la comunidad científica, sino que además son orientadores en la labor profesional y académica, sin embargo, no constituyen en si una verdad definitiva e inmutable, basta con echar una simple mirada histórica a lo acontecido con la Homosexualidad, el TDAH, Autismo, Esquizofrenia, Neurosis, etc. Estos manuales se encuentran en constante revisión, pues solo suponen cierto consenso ligado a un momento y contexto determinados. Por lo que la inclusión de un término o su exclusión en el siguiente manual ha sido parte de un debate permanente que enriquece el proceso ya que la evolución es evidentemente constante.

En esta misma línea surge una directriz de la Organización Mundial de la Salud (OMS) cuyo informe del 18 de junio de 2018, incluye en el CIE-11 los términos de Alienación Parental asociados no como un diagnóstico en sí mismo, sino considerado como sinónimo o un término índice respecto de un diagnóstico específico. Es decir, un problema de relación entre cuidador y niño. El mismo se identifica con el código QE52.0 (CIE-11, 2018) bajo el concepto de Parentalidad implicando problemas asociados a relaciones interpersonales en la niñez. Detalla en su descripción la insatisfacción sustancial y sostenida en la relación entre el cuidador y el niño asociada con perturbación significativa en el funcionamiento. En el índice de términos se especifica: problemas en la relación entre el cuidador y el niño; problemas entre padres y niños; problemas entre cuidador y niño, entre un padre y el cuidador anterior; Alienación Parental; Alejamiento Parental (CIE-11, 2018), lo cual viene a zanjar una parte importante del debate sobre su existencia, no obstante, es perfectible y los manuales deben seguir cambiando y adaptándose a las nuevas situaciones.

Finalmente para los autores Aguilar (2006), Baker y Eichler (2016), Baker y Verrocchio, (2016), Bernet y Baker (2013), Bernet, Baker y Verocchio, (2015), Ferrari (1999), Gardner (1998), Ramírez (2011), Tejedor (2006) y Zicavo (2006), existen una serie de componentes que ayudan a identificar la instauración del proceso de la alienación parental en el transcurso de la ruptura conflictiva de la pareja, siendo los que han guiado la presente investigación pues son los más relevantes, a estos les hemos denominado Componentes para la identificación de la AP los cuales están presentes y son base teórica en la construcción de la Escala ZICAP II.

\section{Componentes para la identificación de la AP}

Indicadores de inicio de manipulación y sugestión maliciosa, en el proceso de alienación. Son conductas de obstrucción inicial -directas o indirectas- al contacto de los hijos con el padre ausente llevadas a cabo por el progenitor que posee la custodia de los hijos. Suele darse a través de una clara y manifiesta manipulación de los afectos y lealtades del niño. Es un proceso sugestivo inicial que intenta volcar la voluntad de la lealtad de los niños por el padre que supuestamente ha sido víctima de la conyugalidad desajustada del otro padre (Aguilar, 2006; Zicavo, 2010).

Uso del tiempo como estrategia de alienación. Cuando uno de los padres se ve forzado a estar ausente de la dinámica familiar por largos períodos temporales, el otro progenitor adquiere gran poder en la formación y dirección de las ideas, creencias, afectos y socialización del niño. La manipulación sistemática sostenida en el tiempo es un arma eficaz en manos del alienador, permite que el hijo se convierta en miembro activo de la campaña de denigración (Tejedor, 2006).

Dificultades en el momento de ejercer el derecho de visita. Se observa cuando (con premeditación) el adulto impide o dificulta las visitas del progenitor ausente con el hijo, programando otras actividades atractivas en ese mismo espacio temporal para que el encuentro se vea frustrado (Zicavo, 2010).

Inmersión judicial. Los progenitores alienadores tienden a usar y abusar de los procesos judiciales y la supervisión de las visitas, interroga a sus hijos sobre lo que han hecho, visto u oído en la convivencia con el otro progenitor, les relata episodios judiciales intentando influir en sus ideas y creencias con la intención de manipular a sus hijos (Ramírez, 2011; Zicavo, 2006).

Campaña de injurias y desaprobación. Producto de la acción sistemática de uno de los padres para volcarlo como aliado en su lucha por responsabilizar al otro de la ruptura conyugal, el 
chico suele contribuir con el proceso alienador de manera activa con injurias y desprecios contra el progenitor distante (Aguilar, 2004; Baker \& Verrocchio, 2016; Gardner, 1998). El niño siente que debe tomar partido en la defensa del progenitor alienador como acto de supuesta justicia (Aguilar, 2004).

Extensión del odio al entorno del progenitor alienado. El niño alienado no sólo muestra antipatía y rechazo al progenitor ausente (sin que haya justificación real para ello) sino a todo lo que a él pueda referirse o a cualquier miembro de la familia de este: abuelos, tíos, primos, etc., personas que sin embargo antes habían mantenido relaciones afectivas cercanas y agradables, de pronto se tornan peligrosas y de las cuales hay que desconfiar o evitar (Aguilar, 2004).

Ausencia de sentimientos de culpa. El niño refiere no sentir culpabilidad alguna por el odio experimentado hacia su padre o madre, ya que el otro lo merece. Constituye un acto de lealtad exigido por el progenitor custodio e incorporado después como conducta libremente asumida. Divide al mundo en buenos y malos, en opuestos irreconciliables sin remordimiento alguno (Aguilar, 2004; Baker \& Verrocchio, 2016).

Fenómeno del "pensador independiente". El niño afirma que la idea de rechazar al progenitor ausente es exclusivamente propia y nadie lo ha influenciado, en clara alusión a una sobre identificación con el alienador custodio (Gardner, 1998). Es indicativo de que la obstrucción relacional está instalada y contribuyendo al desarraigo de los lazos afectivos (Baker \& Verrocchio, 2016; Ferrari, 1999; Zicavo, 2006).

Cuando estos componentes están presentes, indican una nueva forma de maltrato infantil que tendrá efectos psicosociales y devela procesos familiares vinculares de alta conflictividad conducentes a la formación de patrones relacionales disfuncionales en personas que más tarde se insertarán en la sociedad reproduciendo -como tendencia- vínculos familiares desajustados o al menos mecanismos de resolución de conflictos altamente patógenos (ver, por ejemplo, Baker 2005). Aun considerando lo mencionado, no se pueden dejar de lado las discusiones y controversias que el concepto de alienación parental -en tanto síndrome- ha generado en la literatura científica y la práctica clínica desde que fuere acuñado por R. Gardner. En este sentido, como antes referimos, se discuten en la actualidad tanto la denominación del fenómeno como la direccionalidad de este y la implicación de cada actor, entre otras dimensiones (por revisiones, ver, por ejemplo, Kelly \& Johnston, 2001 y Zander, 2012). Más allá de las discusiones, la descripción y caracterización de los vínculos disfuncionales y alienantes durante un divorcio difícil adquieren relevancia científica y clínica en términos de prevenir consecuencias nocivas de esta situación conflictiva relacional (Cunha Gomide, Bedin Camargo \& Gonzales Fernandes, 2016).

\section{Dimensiones de la AP}

En el análisis preliminar de la aplicación del instrumento a una muestra de 257 niños hijos de padres separados (Zicavo et al., 2016), se observó que era posible agrupar los componentes teóricos en tres dimensiones, no obstante, en sucesivos ajustes derivados de aplicaciones instrumentales y análisis estadísticos, se arribó a dos dimensiones como ejes centrales, a través de los cuales se evidencia el proceso de AP (ver Tabla 1), como manifestación del maltrato infantil y la violencia intrafamiliar. Las dimensiones son:

Dimensión I - Conductas Alienantes: captura o secuestro emocional. Comprendida como toda acción consciente de manipulación y sugestión maliciosa llevada a cabo para el logro de compromisos emocionales ineludibles del niño con el progenitor que tiene la guarda y custodia, con quien convive (Zicavo et al., 2016). Sugestiona en la dirección de que solo el tutor que posee la guarda y custodia del niño podrá cuidarlo de manera exitosa, siendo el único merecedor de su cariño, afecto y lealtad. Toda otra persona es sustituible, dejando al hijo capturado, prisionero de una triangulación afectiva y maliciosa (Martínez, 2003; Zicavo et al., 2016). Quienes presentan un mayor nivel de vulnerabilidad e indefensión ante el conflicto paterno, quedan atrapados (amarrados), o son "capturados emocionalmente", convirtiéndose en aliados incondicionales de la 
persona alienadora. Se encuentra comprendida por los componentes: Inicio de manipulación y sugestión; Uso del tiempo como estrategia de alienación o separación; Dificultades en el momento de ejercer el derecho de visita; Inmersión judicial.

Dimensión II - Conductas Alienadas: Evitación, Desprecio y sobre implicación irracional. Para el logro de un ambiente proveedor de seguridad y equilibrio, el niño debe cumplir con las sugestivas demandas de lealtad, injurias, evitación y desprecio a toda persona que no cuente con la aprobación y afecto manifiesto del progenitor custodio. Conduce a un grado superior el proceso de agresión infantil e implica que ha sido involucrado en severas contiendas adultas por espacios de poder (Zicavo et al., 2016). Esta dimensión se encuentra comprendida por los componentes: Campaña de injurias y desaprobación; Extensión del odio al entorno del progenitor alienado; Ausencia de sentimientos de culpa; Fenómeno denominado "pensador independiente".

Tabla 1

Dimensiones, Componentes, Ítems.

\begin{tabular}{|c|c|c|}
\hline Dimensión & Componentes & $\begin{array}{l}\mathrm{N}^{\circ} \text { de } \\
\text { Ítem (29) }\end{array}$ \\
\hline \multirow{4}{*}{$\begin{array}{l}\text { I. Conductas Alienantes Captura o Secuestro } \\
\text { Emocional }\end{array}$} & 1. Inicio de manipulación y sugestión. & 2.10 .19 \\
\hline & $\begin{array}{l}\text { 2. Uso del tiempo como estrategia de } \\
\text { Alienación o separación. }\end{array}$ & 8.25 .28 \\
\hline & $\begin{array}{l}\text { 3. Dificultades en el momento de } \\
\text { ejercer el derecho de visita. }\end{array}$ & 7. 17.24 \\
\hline & 4. Inmersión Judicial. & $\begin{array}{l}9.18 .26 . \\
29\end{array}$ \\
\hline \multirow{4}{*}{$\begin{array}{l}\text { II. Conductas Alienadas Evitación, } \\
\text { Desprecio y sobreimplicación irracional }\end{array}$} & $\begin{array}{l}\text { 5. Campaña de injurias y } \\
\text { desaprobación. }\end{array}$ & $\begin{array}{l}1.11 .20 . \\
27\end{array}$ \\
\hline & $\begin{array}{l}\text { 6. Extensión del odio al entorno del } \\
\text { progenitor alienado. }\end{array}$ & $\begin{array}{l}3.5 .15 . \\
22\end{array}$ \\
\hline & 7. Ausencia de sentimientos de culpa. & $\begin{array}{l}\text { 4. } 12.14 . \\
23\end{array}$ \\
\hline & $\begin{array}{l}\text { 8. Fenómeno denominado "pensador } \\
\text { independiente". }\end{array}$ & $\begin{array}{c}\text { 6. } 13.16 . \\
21\end{array}$ \\
\hline
\end{tabular}

Nota: elaboración propia.

\section{Materiales y Método}

\section{Construcción de la escala de Alienación Parental}

En base a los postulados teóricos, se elaboró un listado de enunciados múltiples dados en proposiciones que operacionalizaron los componentes de la AP. Cada ítem incluyó diversas propuestas a fin de identificar la presencia de cada componente desde diversos ángulos para minimizar la defensa del sujeto. Fueron proposiciones estructuradas y cerradas a través de opciones acotadas en escala tipo Likert de 5 puntos, graduadas según intensidad.

Jueces externos. Su labor consistió en seleccionar -a partir de los 123 reactivos construidos inicialmente-, los más pertinentes para describir la AP. Los expertos también podían incluir ítems no contemplados o realizar modificaciones buscando dar correspondencia entre los elementos teóricos y empíricos del estudio en base a su experiencia. A continuación, se elaboró un registro detallado con aquellos ítems que reunían mayor consenso dando paso a un instrumento preliminar de 87 enunciados. Para evaluar aspectos de comprensión y el tiempo de respuestas de los niños, este cuestionario fue preliminarmente aplicado a un grupo de 13 niños, entre 9 y 15 años, 
estudiantes de un colegio particular subvencionado de la ciudad de Chillán, Chile, todos hijos de padres separados. Al testear la versión de 87 ítems se observó que los niños se cansaron rápidamente $\mathrm{y}$ en oportunidades no comprendieron de manera adecuada algunos enunciados requiriendo apoyo para poder responder. En un proceso de 2 años en sucesivos y cuidadosos pasos de análisis mediante SPSS, de comprensión y tiempos de respuesta (focus group), se procede a eliminar los ítems menos comprendidos y aquellos estadísticamente más débiles hasta arribar a un instrumento más sólido de 48 ítems. Se decidió aplicar la prueba a todos los niños de cada curso escolar que se tuvo acceso, sin diferenciarlos por su condición familiar (hijos de padres separados o no), procedimiento que resulta adecuado a las circunstancias investigativas y que se decide replicar en las siguientes etapas, discriminando posteriormente en base a los datos generales pertinentes.

Prueba Piloto. Se realiza la aplicación piloto de esta versión de 48 ítems a 338 alumnos de otro colegio de la ciudad de Chillán, Chile, y se obtuvieron 123 escalas respondidas por hijos de padres separados. Se observó que el instrumento era respondido aproximadamente entre 10 y 12 minutos y que era de fácil comprensión. Dichas aplicaciones fueron procesadas estadísticamente con el programa Stadistical Package for the Social Sciences (SPSS versión 22). La revisión detallada mediante Alfa de Cronbach de componente por componente e ítem por ítem del instrumento, permitió ir depurándose y eliminar elementos que presentaron baja correlación con el componente respectivo. Dichas consideraciones permitieron el logro de un instrumento de 33 ítems que resultó ser más consistente, siendo a la vez más comprendido y accesible para los niños. Al observar que se había arribado a un instrumento robusto se procedió a aplicarlo a una muestra más amplia de 257 niños, abarcando varias regiones a nivel nacional. Junto lo anterior los investigadores consensuaron en denominarlo Escala Zicap: Para niños de 9 a 15 años (Zicavo et al., 2016).

Aplicación Definitiva. La versión definitiva de la Escala ZICAP II se aplicó a 3385 personas de 7 regiones de Chile, de diversas instituciones que consintieron en incluirse en el proceso. De esas aplicaciones se obtuvieron 1181 cuestionarios respondidos por hijos de padres separados. Las regiones de aplicación del instrumento comprendieron la zona centro y parte del sur de Chile (ver Tabla 2), según el investigador fuera seleccionando directa e intencionadamente las personas de la población mediante los criterios de inclusión de estas y la aceptación consentida expresa.

\section{Población y Muestra}

En el presente estudio se considera población a los niños y adolescentes chilenos hijos de padres separados, con edades comprendidas entre 9 y 15 años. La muestra seleccionada fue de carácter informal, no probabilística, por conveniencia. La muestra estuvo conformada por hijos de padres separados pertenecientes a establecimientos educacionales o centros de mediación familiar de las regiones V a la XVI de Chile. La distribución de la muestra por regiones de Chile, así como edad y sexo, se puede observar a continuación en la Tabla 2, a quienes se aplicó la versión definitiva del instrumento creado. 
Tabla 2

Estadística de la Muestra

\begin{tabular}{lcccccc}
\hline & \multicolumn{2}{c}{ Mujeres } & \multicolumn{2}{c}{ Hombres } & \multicolumn{2}{c}{ Total } \\
& $n$ & $(\%)$ & $n$ & $(\%)$ & $n$ & $(\%)$ \\
\cline { 2 - 7 } Total & 592 & $(50.1)$ & 589 & $(49.9)$ & 1181 & $(100)$ \\
\hline Edad & & & & & & \\
9 & 33 & $(5.6)$ & 42 & $(7.1)$ & 75 & $(6.4)$ \\
10 & 77 & $(13.0)$ & 87 & $(14.8)$ & 164 & $(13.9)$ \\
11 & 114 & $(19.3)$ & 115 & $(19.5)$ & 229 & $(19.4)$ \\
12 & 126 & $(21.3)$ & 110 & $(18.7)$ & 236 & $(20.0)$ \\
13 & 115 & $(19.4)$ & 76 & $(12.9)$ & 191 & $(16.2)$ \\
14 & 67 & $(11.3)$ & 88 & $(14.9)$ & 155 & $(13.1)$ \\
15 & 50 & $(8.4)$ & 64 & $(10.9)$ & 114 & $(9.7)$ \\
16 & 10 & $(1.7)$ & 7 & $(1.2)$ & 17 & $(1.4)$ \\
\hline Región & & & & & & \\
Valparaíso & 121 & $(20.4)$ & 117 & $(19.9)$ & 238 & $(20.2)$ \\
Santiago & 95 & $(16.0)$ & 98 & $(16.6)$ & 193 & $(16.3)$ \\
O’Higgins & 11 & $(1.9)$ & 12 & $(2.0)$ & 23 & $(1.9)$ \\
Maule & 27 & $(4.6)$ & 29 & $(4.9)$ & 56 & $(4.7)$ \\
Nuble & 150 & $(25.3)$ & 151 & $(25.6)$ & 301 & $(25.5)$ \\
Bío Bío & 86 & $(14.5)$ & 80 & $(13.6)$ & 166 & $(14.1)$ \\
Los Lagos & 102 & $(17.2)$ & 102 & $(17.3)$ & 204 & $(17.3)$ \\
\hline
\end{tabular}

\section{Consideraciones Éticas y Proceso de Obtención de la Información}

Para la presente investigación se resguardaron las consideraciones éticas a través de diversos procedimientos. Por una parte, el consentimiento, asentimiento informado y la revisión de la investigación fueron presentados al comité de ética de la universidad de referencia y se aseguró la confidencialidad de los datos, lo que implicó la protección de toda información considerada personal, comunicada entre personas (França-Tarragó, 1996). No se solicitaron datos de identidad de los participantes, sino de referencia general y aplicación, tales como, edad, género, convivencia y estado civil de los padres. Una vez que se contó con el visto bueno de todas las personas implicadas en el proceso incluyendo los padres o apoderados de los niños (conocimiento, comprensión, aceptación), y de los niños mismos, se pasó a las aplicaciones respectivas, proceso muy largo (meses) y engorroso, pero altamente necesario.

\section{Resultados}

Para llevar a cabo el análisis factorial exploratorio (AFE) y posteriormente el análisis factorial confirmatorio (AFC), se dividió al azar la muestra total, tomando solo 274 casos para el análisis de AFE quedando los 907 restantes para el AFC. Con el fin de analizar si los datos cumplían los requisitos para aplicar este procedimiento se estimó la medida de Adecuación Muestral de Kaiser-Meyer-Olkin que entrega un valor de .866; y también se aplicó la prueba de Esfericidad de Bartlett que arroja un resultado de $\chi^{2}(g l=528)=3596.87 p<.0001$. Por otra parte, para analizar la normalidad de los datos se estimaron los valores de asimetría y curtosis para cada uno de los ítems, los que indicaron que solo 7 de ellos se acercan a la distribución normal y 26 ítems muestran una distribución que se aleja de la curva normal. Dado que los datos presentan condiciones que permiten realizar un análisis factorial, pero no se observa normalidad univariada ni multivariada, se utilizó el procedimiento de mínimos cuadrados no ponderados para realizar los 
Análisis Factoriales Exploratorio y Confirmatorio; y como se espera que los factores estén correlacionados en el AFE, se utilizó el método de rotación Oblimin. Como procedimiento adicional se estimó la correlación anti-imagen, a partir de la cual se eliminaron los ítems con valores inferiores a 0.8 obteniendo un instrumento final de 29 ítems, sobre el cual se realizan los siguientes análisis.

Se realiza el AFE sobre los 29 ítems con la muestra de 274 sujetos (Tabla 3) lo cual confirma la propuesta teórica de dos dimensiones. Posteriormente se hace el AFC con la muestra de 907 niños cuyos resultados se muestran en Figura 1. Se considera un buen ajuste del modelo cuando $\chi^{2}$ no es significativo, sin embargo, esta prueba es sensible al tamaño muestral tendiendo a ser significativo en casos como el de este estudio con un elevado tamaño muestral ( $n=907$ casos), por ello se consideraron otras medidas de bondad de ajuste tales como el $\chi^{2}$ dividido por los grados de libertad que debe ser cercano a cero, pero se consideran valores aceptables menores a 5 . Asimismo, los índices GFI, AGFI y CFI, todos los cuales deben ser mayores a .95 y el RMSEA debe ser cercano a cero o menor a .05 (Escobedo, Hernández, Estebané \& Martínez, 2016; Pérez $\&$ Medrano, 2010). En los resultados obtenidos para el AFC, se observa un $\chi^{2}(g l=376)=979.06$ el que es significativo $(p<.0001)$; un $\chi^{2} / g l=2.6$; el GFI= .969; el AGFI= .964; el CFI= .901; y el $\mathrm{RMSEA}=.042$. Por lo anterior, es posible afirmar que el modelo testeado muestra valores de ajuste aceptables, con los datos obtenidos en la muestra de hijos de padres separados.

Tabla 3

Matriz de estructura, $n=274$

\begin{tabular}{ccc}
\hline Ítems & \multicolumn{2}{c}{ Factor } \\
\cline { 2 - 3 } & 1 & 2 \\
\hline 1 & $\mathbf{. 4 7 6}$ & .228 \\
2 & .241 & $\mathbf{. 4 1 0}$ \\
3 & $\mathbf{. 4 5 9}$ & .343 \\
4 & $\mathbf{. 6 8 1}$ & .143 \\
5 & $\mathbf{. 5 4 8}$ & .333 \\
6 & $\mathbf{. 7 7 5}$ & .578 \\
7 & .426 & $\mathbf{. 4 2 1}$ \\
8 & .282 & $\mathbf{. 6 6 6}$ \\
9 & .257 & $\mathbf{. 4 2 3}$ \\
10 & .418 & $\mathbf{. 5 1 2}$ \\
11 & $\mathbf{. 6 4 6}$ & .369 \\
12 & $\mathbf{. 6 1 7}$ & .460 \\
13 & $\mathbf{. 7 3 9}$ & .300 \\
14 & $\mathbf{. 4 8 0}$ & .334 \\
15 & $\mathbf{. 4 8 2}$ & .345 \\
16 & $\mathbf{. 6 0 5}$ & .299 \\
17 & .236 & $\mathbf{. 6 9 4}$ \\
18 & .360 & $\mathbf{. 5 0 7}$ \\
19 & .420 & $\mathbf{. 7 0 0}$ \\
20 & $\mathbf{. 4 2 5}$ & .468 \\
21 & $\mathbf{. 6 4 9}$ & .341 \\
22 & $\mathbf{. 4 6 0}$ & .466 \\
23 & $\mathbf{. 6 8 4}$ & .446 \\
24 & .365 & $\mathbf{. 4 0 8}$ \\
25 & $\mathbf{. 6 8 8}$ & .386 \\
26 & .370 & $\mathbf{. 7 1 0}$ \\
27 & $\mathbf{. 3 6 4}$ & .331 \\
28 & .359 & $\mathbf{. 7 1 4}$ \\
29 & .349 & $\mathbf{4 0 8}$ \\
\hline & &
\end{tabular}




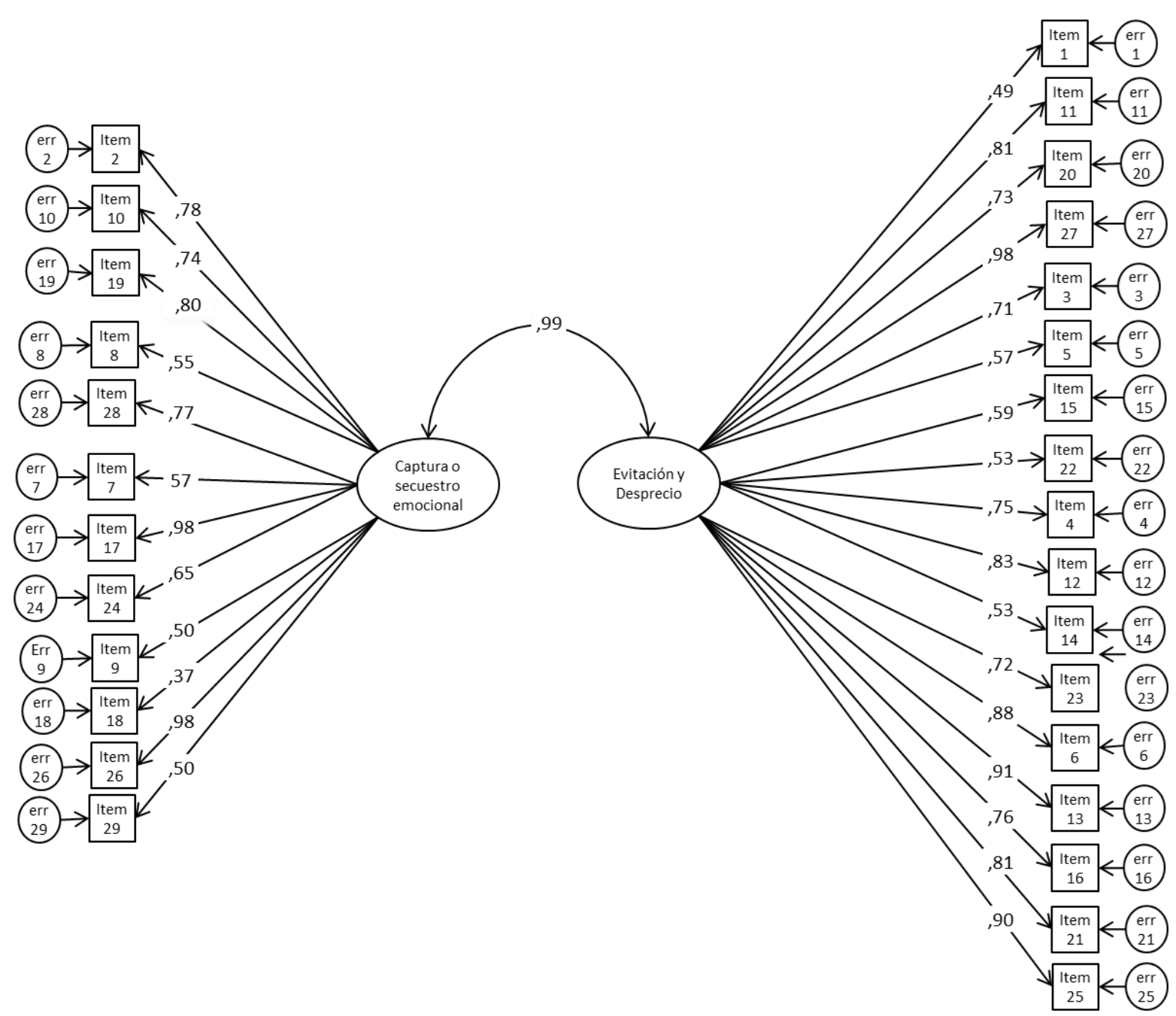

Figura 1. Análisis factorial confirmatorio, $n=907$

Se analizó la confiabilidad en cada una de las dimensiones utilizando el coeficiente Alfa de Cronbach. Se observa que, en la Dimensión I, Conductas Alienantes captura o secuestro emocional, los doce ítems que la componen arrojan un alfa de .777, dicho valor disminuiría al eliminar cualquiera de los ítems. Respecto de la Dimensión II, Conductas Alienadas Evitación, Desprecio y sobreimplicación irracional, indica un alfa de .884 para los diecisiete ítems que la componen y este valor bajaría al eliminar cualquiera de los ítems. Finalmente, se construyeron los baremos de la Escala ZICAP II y de cada una de las dimensiones considerando los promedios y las desviaciones típicas observados en la muestra para estimar los puntajes estandarizados

Para obtener los valores estandarizados se realizaron comparaciones según valores totales de la escala y de cada una de las dimensiones según sexo y edad y no se observan diferencias significativas por lo que las tablas de valores estandarizados se calculan a partir de los datos de toda la muestra. Finalmente, se construyeron los baremos de la Escala ZICAP II y de cada dimensión, considerando los promedios y las desviaciones típicas observados en la muestra para estimar los puntajes estandarizados, con un promedio de 50 y una desviación 20.

Los puntajes estandarizados inferiores a 90 se consideran Ausencia de AP; puntajes iguales o mayores a 90 y menores a 110 se consideran Ligeros AP; puntajes estandarizados iguales o superiores a 110 e inferiores a 130 se consideran AP moderados; y las puntuaciones estandarizadas iguales o superiores a 130 se consideran AP graves. La discusión adicional relacionada con la clasificación mencionada anteriormente se encontrará en la siguiente sección. 


\section{Discusión y Conclusiones}

La validez de los resultados obtenidos con la Escala ZICAP II está respaldada por el constructo teórico subyacente a los fenómenos estudiados pues se resguardó la creación, adaptación y validación de los diversos componentes que dan cuenta de la solidez del instrumento.

Los resultados obtenidos en el análisis realizado indican que los modelos procesados por el análisis factorial confirmatorio presentan niveles de ajuste aceptables, lo que confirma una estructura factorial teórica dividida en dimensiones centrales y componentes integrantes que resulta diferente a la propuesta inicialmente en el artículo Escala Zicap para la evaluación de la alienación parental: resultados preliminares (Zicavo et al., 2016). La diferencia radica en los sucesivos ajustes realizados a partir de los análisis estadísticos con los datos obtenidos lo cual arroja una solución más parsimoniosa para el abordaje de la propuesta teórica, con un instrumento más ajustado, esta vez denominado Escala ZICAP II.

Por otra parte, el análisis estadístico de la matriz estructural permite confirmar la validez de la propuesta teórica inicial de dos dimensiones y 8 componentes, no obstante, se observa que los ítems 7, 20 y 22 contribuyen con cargas similares en ambos factores, decidiéndose mantenerlos en los factores asignados por la teoría. Esto es posible de explicar por la alta correlación existente entre los factores, pues ambos son parte inherente del proceso de AP. La Escala ZICAP II presenta adecuada consistencia interna, lo que permite afirmar que es un instrumento estadísticamente confiable.

A partir de las aplicaciones realizadas se observa que un $17.6 \%$ de los casos evidencian presencia de AP, donde la mayoría, un $15.3 \%$ muestra un nivel de AP Leve, el 1.9\% AP Moderada y un $0.4 \%$ de AP Severa. Los niños que obtienen altos puntajes en la escala indicarían presencia de AP, manteniendo correspondencia con los postulados teóricos que evidencian el proceso de maltrato infantil. Los casos detectados con presencia de AP parecen coincidir con la línea reportada en la literatura existente y otros estudios relacionados últimamente (Baker \& Eichler, 2016; Baker \& Verrocchio, 2016; Bernet \& Baker, 2013; Bernet et al., 2015), estos niños manifiestan un elevado rechazo a contactarse con el progenitor con quien no viven, así como una imagen muy deteriorada del padre y su entorno familiar a quien entienden como completamente malos y prescindibles en su vida, constituyendo evidencias de un acto violento, de agresión que causa profundo daño emocional, un tipo de maltrato y violencia aún no contenido en los manuales de violencia intrafamiliar actuales.

Con la estandarización de la Escala ZICAP II se puede determinar la ausencia de AP en aquellos puntajes bajos. Esto constituye un elemento de trascendental importancia, ya que puede permitir a los especialistas contar con un instrumento eficiente para demostrar con evidencias, la veracidad objetiva de sus pericias en el ámbito clínico, social y jurídico. De esta manera puede contribuir a minimizar posibles errores periciales-diagnósticos que, de lo contrario, tendrían graves consecuencias para la salud psicológica de los niños y sus familias.

El punto de corte para los grados de AP es principalmente estadístico, aun no hay suficientes criterios clínicos para respaldar tal gradualidad, no obstante, se asume cierta correspondencia con los criterios teóricos que se detallan a continuación:

AP Leve. etapa en la cual las visitas paternas al hijo transcurren con algunas limitaciones intencionales, si bien el conflicto es limitado, la tensión se encuentra presente (Gardner, 2002). El hijo suele poseer un pensamiento y emocionalidad independiente, aunque respalde puntualmente al padre alienador (Aguilar, 2006).

AP Moderado. Etapa donde aumentan significativamente los conflictos y litigios entre progenitores (Gardner, 2002). La campaña de menosprecio se acentúa, aumentan los intentos de anulación del rol o desparentalización (Ramírez, 2011). Los lazos afectivos con el padre no 
custodio se deterioran progresiva y profundamente mientras que se intensifican lazos de dependencia emocional manipuladora con el padre custodio.

AP Severo. Etapa en que las acciones de desprestigio son extremas y continuas. Los encuentros con el padre no custodio se hacen imposibles (Gardner, 2002). El odio del hijo hacia el progenitor alienado es extremo con ausencia de culpa (Aguilar, 2006). Es común que el padre alienado se vea impedido de ver a sus hijos por meses, años.

Todo lo anterior evidencia que el instrumento resulta adecuado para medir AP en niños de 9 a 15 años del centro sur de Chile, hijos de padres separados. La Escala ZICAP II es una herramienta de medición confiable y constituye, junto a otros trabajos (Cunha et al., 2016), uno de los primeros intentos en Latinoamérica de dar cuenta empíricamente del fenómeno de la alienación parental. Estudios posteriores podrían evaluar el potencial aplicado de la herramienta desarrollada en el presente estudio, dirigiendo la mirada a la prevención de la violencia intrafamiliar durante el proceso del divorcio difícil. Las proyecciones aplicadas de la escala son, en este sentido, muy alentadoras.

La Escala ZICAP II no constituye en sí mismo un instrumento acabado y cerrado, es plausible que en un futuro cercano sea aún renovado. Requiere de otras miradas, demanda el incesante perfeccionamiento de colegas empeñados en desterrar el maltrato infantil en defensa de los derechos de los hijos de padres separados.

Por último, es importante señalar que este instrumento pretende incorporar un enfoque basado en los derechos de los niños, niñas y adolescentes (NNA) a ser considerados y tenidos en cuenta, a ser reconocidos como titulares de derechos y no como beneficiarios de la benevolencia de los adultos (Pautassi \& Royo, 2012), de ahí la importancia en desarrollar instrumentos que consideren su voz, su testimonio ante divorcios altamente conflictivos.

\section{Recomendaciones y limitaciones}

Aunque el alcance de este trabajo está respaldado por un estudio de 4 años de duración de más de tres mil trescientos ochenta casos, de los cuales mil ciento ochenta y uno eran hijos de padres separados, en siete regiones del centro y sur de Chile, es importante validar la escala ZICAP II en otros contextos culturales y en diferentes países, contribuyendo a su escala y estandarización para otras naciones y poblaciones. Esa es una tarea pendiente que seguramente podrán abordar los colegas interesados en el tema. Del mismo modo, es importante expandir los estudios a otros segmentos de edad de los NNA, validando la Escala ZICAP II y corrigiendo posibles distorsiones y sesgos que puedan estar presentes en este estudio.

\section{Referencias}

Aguilar, J.M. (2004). S.A.P; Síndrome de Alienación Parental. España: Almuzara.

Aguilar, J.M. (2006). Con papá y con mamá. España: Almuzara.

Baker, A. (2005) The Long-Term Effects of Parental Alienation on Adult Children: A Qualitative Research Study. The American Journal of Family Therapy, 33, 289-302.

Baker, A. \& Eichler, A. (2016). The Linkage Between Parental Alienation Behaviors and Child Alienation. Journal of Divorce \& Remarriage, 57(7), 475-484. doi: 10.1080/10502556.2016.1220285

Baker, A. \& Verrocchio, M.C. (2016). Exposure to Parental Alienation and Subsequent Anxiety and Depression in Italian Adults. The American Journal of Family Therapy, 44(5), 255271. doi: 10.1080/01926187.2016.1230480

Bernet, W. \& Baker, A. (2013). Parental alienation, DSM-5, and ICD-11: Response to critics. The journal of the American Academy of Psychiatry and the Law, 41(1), 98-104. 
Bernet, W., Baker A. \& Verrocchio M.C. (2015). Symptom Checklist-90-Revised Scores in Adult Children Exposed to Alienating Behaviors: An Italian Sample. Journal of Forensic Sciences, 60, 357-362. doi: 10.1111/1556-4029.12681

Bernet, W., Wamboldt, M. Z., \& Narrow, W. E. (2016). Child Affected by Parental Relationship Distress. Journal of the American Academy of Child and Adolescent Psychiatry, 55(7), 571-579. doi: 10.1016/j.jaac.2016.04.018

CIE-11 (2018). Clasificación Internacional de Enfermedades, OMS. Recuperado de https://icd.who.int/browse11/1m/en\#/http\%3a\%2f\%2fid.who.int\%2ficd\%2fentity\%2f547677013

Cunha Gomide, P.I., Bedin Camargo, E. \& Gonzales Fernandes, M. (2016) Analysis of the Psychometric Properties of a Parental Alienation Scale. Paidéia, 26(65), 291-298.

Escobedo, M.T.; Hernández, J.; Estebané, V. \& Martínez, G. (2016). Modelos de ecuaciones estructurales: características, fases, construcción, aplicación y resultados. Ciencia y Trabajo, 18(55), 16-22.

Ferrari, J.L. (1999). Ser padres en el tercer milenio. Argentina: Canto Rodado.

Folberg, J. \& Milne, A. (2005). Divorcio como posible etapa del ciclo vital o divorcio destructivo. Revista de psicoterapia relacional e intervenciones sociales, 15, 18-24.

França-Tarragó, O. (1996). Ética para psicólogos. Introducción a la Psicoética. Bilbao: Desclée de Brouwer.

Gardner, R. (1998). The Parental Alienation Syndrome (2n ed.). Cresskill, New Jersey: Creative Therapeutics.

Gardner, R. (2002). Denial of the Parental Alienation Syndrome Also Harms Women. American Journal of Family Therapy, 30(3), 191-202. doi: 10.1080/019261802753577520

Harman, J.J., Biringen, Z., Ratajack, E.M., Outland, P.L. \& Kraus, A. (2016). Parents Behaving Badly: Gender Biases in the Perception of Parental Alienating Behaviors. Journal of Family Psychology, 30(7), 866-874. doi: 10.1037/fam0000232

Kelly, J.B. \& Johnston, J.R. (2001). The Alienated Child. A Reformulation of Parental Alienation Syndrome. Family Court Review, 39(3), 249-266. doi: 10.1111/j.174-1617.2001.tb00609.x

Lesme, D. \& Zicavo, N. (2014). Hijos que viven “entre” la casa de mamá y la de papá. Integración Académica en Psicología, 2(5), 21-28.

Ley $\mathrm{N}^{\circ} 20.066$ (2005). Ley de violencia Intrafamiliar. Recuperado de: http://www.leychile.cl/Navegar?idNorma=242648

Ley $\mathrm{N}^{\circ} 20.480$ (2010). Ley de Femicidio. Recuperado de: http://www.bcn.cl/de-que-sehabla/promulgacion-femicidio

Ley N 20.680 (2013). Ministerio de Justicia de Chile. Ley Amor de Papá. Biblioteca del Congreso Nacional de Chile. Recuperado de: https://www.leychile.cl/Navegar?idNorma=1052090\&buscar=Ley+20680

Martínez, R. L. (2003). El conflicto en la vida de la pareja. En O. P. Salud, La familia, su dinámica y tratamiento (pp. 122-124). Washington DC: Instituto Mexicano de Seguridad Social.

Moses, M., \& Towsend, B. A. (2011). Parental Alienation in child custody disputes. Tennesee Bar Journal. 47(5), 25-29

O'Donohue, W., Benuto, L.T. \& Bennett, N. (2016). Examining the validity of parental alienation syndrome. Journal of Child Custody, 13 (2-3), 113-125. doi: 10.1080/15379418.2016.1217758

Pautassi, L. \& Royo, L. (2012). Enfoque de derechos en las políticas de infancia: indicadores para su medición. CEPAL, UNICEF. Recuperado de: http://www.derecho.uba.ar/investigacion/investigadores/publicaciones/pautassienfoquedederechosenlaspoliticasdeinfancia.pdf

Pepiton, M. B., Alvis, L. J., Allen, K., \& Logid, G. (2012). Is parental alienation disorder a valid concept? Not according to scientific evidence: A review of parental alienation, DSM-5 and 
ICD-11 by William Bernet. Journal of Child Sexual Abuse, 21(2), 244-253. doi: 10.10 80/10538712.2011.628272

Pérez, E. \& Medrano, L. (2010). Análisis factorial exploratorio: bases conceptuales y metodológicas. Ciencias del Comportamiento, 2(1), 58-66.

Ramírez, D. (2011). Las prácticas de desparentalización que se les imponen a los hombres, tras la separación o el divorcio de su pareja: posibles secuelas psicosociales, para ellos y su prole. Estudio de casos, peritados y dictaminados en el año 2008, para el Juzgado de Familia del Segundo Circuito Judicial. Facultad de Ciencias Humanas, Universidad Autónoma de Centro América, San José.

Siracusano, A., Barone, Y., Lisi, G., \& Niolu, C. (2015). Parental alienation syndrome or alienating parental relational behaviour disorder: a critical overview. Journal of Psychopathology, 21, $231-238$

Tejedor, M. (2006). El síndrome de alienación parental: una forma de maltrato (2a ed.). Madrid: EOS.

Zander, J. (2012). Parental Alienation as an Outcome of Paternal Discrimination. New Male Studies: An International Journal, 1(2), 49-62.

Zicavo, N. (2006). ¿Para qué sirve ser Padre? Concepción: Ediciones Universidad del Bio-Bio.

Zicavo, N. (2010). Crianza Compartida. Síndrome de Alienación Parental, Padrectomía, los Derechos de los Hijos ante la Separación de los Padres. México: Trillas.

Zicavo, N. (2016). Parentalidad y Divorcio. (Des)encuentros en la familia Latinoamericana. México, Alfepsi Editorial.

Zicavo, N., Celis, D., González, A., \& Mercado, M. (2016). Escala Zicap para la evaluación de la alienación parental: resultados preliminares. Ciencias Psicológicas, 10(2), 177-187.

Zirogiannis, L. (2001). Evidentiary issues with Parental Alienation Syndrome. Family Court Review, 39(3), 334-343. doi: 10.1111/j.174-1617.2001.tb00614.x

Contribución de los autores: a) Concepción y diseño del trabajo; b) Adquisición de datos; c) Análisis e interpretación de datos; d) Redacción del manuscrito; e) revisión crítica del manuscrito. N. Z. M. ha contribuido a, b, c, d, e; R. R. C. en c, e; L. P. en e.

Editora científica responsable: Dra. Cecilia Cracco 